\title{
APPROCHE DE DÉTERMINATION DE SIGNATURE DE TEXTURE - APPLICATION À LA CLASSIFICATION DE COUVERTS FORESTIERS D'IMAGE SATELLITAIRE À HAUTE RÉSOLUTION
}

\author{
Wala Zaaboub ${ }^{1}$, Zouhour Ben Dhiaf ${ }^{1}$ \\ 1: Laboratoire d'Informatique Programmation Algorithmique et Heuristique, FST Campus Universitaire, 2092 El Manar, Tunis-Tunisie \\ walazaaboub@gmail.com, zouhour_bendhiaf@yahoo.fr
}

\begin{abstract}
Résumé
Cet article présente une approche d'identification de signature de texture pour la classification d'image satellite à haute résolution de scènes forestières. Nous cherchons la combinaison la plus adéquate d'attributs issus de mesures de texture. Cette combinaison d'attributs, qui forme notre signature, doit permettre la discrimination entre les différents types de textures présents dans l'image à classer. Nous améliorons notre signature par une étape de pondération des attributs. Le poids de chaque attribut traduit son degré de confiance. Nous terminons par une étape d'expérimentation consistant à appliquer notre signature combinée pondérée pour des fins de classification d'image satellite haute résolution de zones forestières.
\end{abstract}

Mots clés : Signature de texture, classification, histogrammes de sommes et de différences, image satellite haute résolution, multifractal, espèces forestières.

\begin{abstract}
This paper presents an approach for identifying textural signature for the classification of high resolution satellite image of forest. We are looking for the most appropriate combination of features from texture measures. This combination of features, which forms our signature, should allow the discrimination between different types of textures present in the image that will be classified. We improve our signature by a step of feature weighting. The weight of each feature reflects its degree of confidence. We finish with an experimental step which is an application of our combined weighted signature for the purposes of classification of high resolution satellite image of forests.
\end{abstract}

Keywords : Texture signature, classification, sum and difference histogram, high resolution satellite image, multifractal, forest species.

\section{Introduction}

Malgré la diversité des méthodes de classification qui servent à fournir à partir d'une image satellite une cartographie de l'occupation du sol, plusieurs problèmes persistent.

La problématique générale de cet article est la classification des différents couverts forestiers, qui s'intègre dans une problématique écologique et économique.

Au cœur de cette problématique, l'objectif de notre étude est d'obtenir une cartographie automatique ou semi-automatique des espèces d'arbres forestiers. Cette cartographie doit aussi séparer les zones forestières et les zones non couvertes par les arbres. La gestion des ressources forestières est critique pour notre zone d'étude en raison de la biodiversité qu'elle abrite.

Pour les méthodes de classification spectrale, l'information radiométrique des images satellitaires s'avère incapable de distinguer les occupations du sol ayant des caractéristiques radiométriques identiques.

C'est dans ce cadre que s'intègre cet article dédié à la classification basée texture d'image satellitaire haute résolution de scène forestière.

La texture est définie par des descripteurs qualitatifs appelés "mesures de texture" (ex. finesse) qui sont formalisées par des descripteurs quantitatifs appelés "attributs de texture" (ex. entropie). Pour cela nous notons la nécessité d'utiliser une "signature de texture" dans le processus de classification. Nous définissons notre signature comme un vecteur d'attributs permettant de faire la discrimination entre les différentes textures présentes dans l'image.

Dans la littérature, la plupart des chercheurs effectuent un grand nombre des tests préliminaires afin de déterminer la signature de texture convenable (Marceau et al., 1990 ; Messier et al., 2001 ; Akono et al., 2003). Ces tests consistent à faire des classifications basées texture, en utilisant des signatures candidates construites par des combinaisons des attributs de texture extraits à partir d'une méthode d'analyse. Ainsi, la signature optimale est la combinaison des attributs de texture permettant d'obtenir un taux de classification maximal. Cependant, notre approche d'identification de signature 


\begin{tabular}{|l|c|c|c|c|}
\hline Critères Méthodes & $\begin{array}{c}\text { Histogramme de } \\
\text { niveaux de gris }\end{array}$ & $\begin{array}{c}\text { Matrice de } \\
\text { cooccurrence }\end{array}$ & $\begin{array}{c}\text { Histogrammes de somme } \\
\text { et de différence }\end{array}$ & $\begin{array}{c}\text { Statistiques d'ordre } \\
\text { supérieur à deux }\end{array}$ \\
\hline $\begin{array}{l}\text { Statistiques sur la } \\
\text { disposition spatiale }\end{array}$ & Non & Oui & Oui & Très élevé \\
\hline Temps de calcul & Faible & Elevé & Moyen & Très élevé \\
\hline $\begin{array}{l}\text { Espace mémoire util- } \\
\text { isé }\end{array}$ & Faible & Elevé & Moyen & Elevé \\
\hline $\begin{array}{l}\text { Nombre de } \\
\text { paramètres à définir }\end{array}$ & Faible & Elevé & Elevé & Non \\
\hline $\begin{array}{l}\text { Perte d'information } \\
\text { spectrale }\end{array}$ & Non & Oui & Oui \\
\hline
\end{tabular}

TABLE 1 : Synthèse des comparaisons des méthodes d'analyse statistique.

consiste tout d'abord à déterminer des mesures de texture "formant" la signature sans avoir recours à l'étape de tests. Après, nous proposons les attributs de texture qui peuvent représenter notre signature. Ensuite, nous déterminons (à l'aide d'un processus que nous proposerons) notre combinaison de certains attributs en considérant les mesures déterminées et les méthodes d'analyse de texture les plus adéquates pour notre contexte.

La suite de cet article est organisée de la manière suivante : la section 2 détaille la première étape de notre approche d'identification de signature en exposant notre synthèse des comparaisons des méthodes d'analyse de texture, en tenant compte de notre type de données. La section 3 illustre particulièrement les méthodes d'analyse utilisées dans notre approche. La section 4 détaille la deuxième étape qui sert à déterminer les mesures de texture pour la signature, et les attributs utilisés dans le processus proposé. La section 5 présente la troisième étape qui est le processus d'identification de signature combinée. La section 6 détaille la dernière étape qui sert à déterminer des poids pour les attributs de notre signature. Les résultats obtenus sont détaillés à la section 7 . Enfin la section 8 présente la conclusion de cette étude.

\section{Synthèse des comparaisons des méthodes d'analyse de texture}

La première étape de l'approche proposée de détermination de signature de texture consiste à faire une synthèse des comparaisons par catégorie des méthodes d'analyse de texture.

Cette comparaison est réalisée à l'aide des points forts et des points faibles des méthodes les plus utilisées, après une recherche dans plusieurs travaux (Gabor, 1946; Haralick et al., 1973; Galloway, 1975; Unser, 1986 ; Ojala et al., 1996 ; Diou, 2000 ; Rellier et al., 2002 ; Grazzini, 2003 ; Abadi, 2008 ; Porebski, 2010 ; Prasetiyo, 2010; Tan and Triggs, 2010; Lefebvre et al., 2011; Djema et al., 2012 ; Penatti et al., 2012).

De plus, nous avons déduit d'autres propriétés à partir des comparaisons apparaissant dans des travaux de recherche antérieures. À noter que ces comparaisons ne sont pas faites par catégorie, ce qui est la raison de notre synthèse.

Parmi les critères indispensables pour le choix de la méthode d'analyse adéquate pour notre tâche et notre type de texture, nous citons:

- Une caractérisation des textures irrégulières, parce que notre texture est naturelle et non régulière;

- Une complexité acceptable, car nous utilisons une image satellitaire à haute résolution ;

- Une analyse multi-résolution, pour augmenter la capacité à discriminer les textures ;

- Une caractérisation locale de texture, puisque nous nous intéressons à la localisation des espèces forestières;

- Un faible nombre de paramètres.

Nous résumons les critères de chaque catégorie de méthodes dans trois tableaux récapitulatifs (cf. Tableaux 1,2 et 3 ).

Pour la catégorie des méthodes d'analyse statistique, la méthode des matrices de cooccurrence (M.C.O) (Haralick et al., 1973) et la méthode des histogrammes de sommes et de différences (H.S.D) (Unser, 1986) nous semblent les plus adéquates (cf. Tableau 1). Cependant, nous choisissons la méthode des H.S.D puisqu'elle est plus rapide que la méthode des M.C.O avec presque la même capacité de discrimination de texture. Pour la catégorie de méthodes d'analyse fréquentielle, la méthode de filtre de Gabor (Gabor, 1946) et la méthode de transformée d'ondelette nous semblent les plus fructueuses (cf. Tableau 2).

Toutefois, nous choisissons la méthode de transformée en ondelettes car son temps de calcul est plus faible et elle ne nécessite pas un grand nombre de paramètres à régler. Concernant la catégorie de méthodes d'analyse basée sur la modélisation de texture, les méthodes basées sur les modèles multifractals et les méthodes basées motifs ternaires locaux (LTP) (Tan and Triggs, 2010) nous semblent les plus appropriées (cf. Tableau 3). Mais, puisque nous ne sommes pas concerné de caractériser uniquement les petits détails, la méthode multi- 


\begin{tabular}{|c|c|c|c|c|}
\hline Critères Méthodes & Transformée de Fourier & Transformée de Gabor & Filtre de Gabor & Transformée en ondelettes \\
\hline $\begin{array}{l}\text { Représentation spa- } \\
\text { tiale + fréquentielle }\end{array}$ & Non & Oui & Oui & Oui \\
\hline Multi-résolution & Non & Non & Oui & Oui \\
\hline Temps de calcul & Moyen & Elevé & Elevé & Faible \\
\hline $\begin{array}{l}\text { Caractérisation de } \\
\text { texture }\end{array}$ & Globale & Globale et locale & Globale et locale & Globale et locale \\
\hline $\begin{array}{ll}\text { Nombre } & \text { de } \\
\text { paramètres à définir }\end{array}$ & Faible & Elevé & Elevé & Faible \\
\hline
\end{tabular}

TABLE 2 : Synthèse des comparaisons des méthodes d'analyse fréquentielle.

fractale est la méthode la plus adéquate pour notre cas.

\section{Méthodes d'analyse de texture utilisées}

Parmi les trois méthodes d'analyse de texture les plus adéquates pour notre contexte (cf. Section 2), nous utilisons seulement deux méthodes pour notre approche d'identification de signature. Ce choix est argumenté dans la section suivante (cf. Section 4.). Pour des raisons de clarté et d'économie d'espace, nous présentons uniquement les méthodes d'analyse utilisées dans notre approche ainsi que leurs indices.

\subsection{Méthode d'analyse des histogrammes de sommes et de différences}

La méthode des histogrammes de sommes et de différences (H.S.D) d'Unser (1986) est une technique alternative pour calculer un bon nombre d'attributs d'Haralick et al. (1973) sans avoir recours aux matrices de cooccurrence. Prenant une image $I$ représentée par une matrice (dimension $=K \times L)$ dont les éléments sont $y_{k, l}$ avec $k=0 . . K-1 ; l=0 . . L-1$. Les H.S.D sont définis de la manière suivante :

$$
\left\{\begin{array}{l}
h_{s}(i ; d 1, d 2)=h_{s}(i)=\operatorname{Card}(k, l) \in I, s_{k, l}=i \\
h_{d}(j ; d 1, d 2)=h_{d}(i)=\operatorname{Card}(k, l) \in I, d_{k, l}=j
\end{array}\right.
$$

Avec :

$$
\left\{\begin{array}{r}
s_{k, l}=y_{k, l}+y_{k+d 1, l+d 2} \\
d_{k, l}=y_{k, l}-y_{k+d 1, l+d 2}
\end{array}\right.
$$

À noter que le couple $(d 1, d 2)$ dépend du couple de paramètres $(d, p)$; avec $d$ la direction d'échantillonnage et $p$ le pas d'échantillonnage (cf. Figure 1).

L'algorithme des H.S.D utilise deux vecteurs normalisés de dimension $\left[2 N_{g}-1\right]\left(N_{g}\right.$ : le nombre de niveaux de gris dans l'image $I$ ) formés par des éléments $\hat{P}_{s}(i)$ et $\hat{P}_{d}(j)$ définis comme suit :

$$
\begin{cases}\hat{P}_{s}(i)=\frac{h_{s}(i)}{N} ; \quad & \left(i=1, . ., 2 N_{g}-1\right) \\ \hat{P}_{d}(j)=\frac{h_{d}(j)}{N} ; \quad\left(j=-N_{g}+1, . ., N_{g}-1\right) \\ N=\sum_{i} h_{s}(i)=\sum_{j} h_{d}(j)\end{cases}
$$

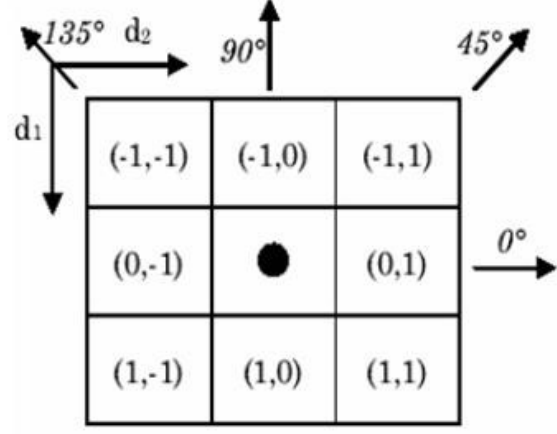

FIGURE 1 : Quelques valeurs possibles du couple $(d 1, d 2)$.

Où $\hat{P}_{s}(i)$ et $\hat{P}_{d}(j)$ sont les estimateurs des fonctions de probabilité de la somme et de la différence :

$$
\left\{\begin{array}{l}
P_{s}(i)=\operatorname{proba}\left\{s_{k, l}=i\right\} ;\left(i=1, . ., 2 N_{g}-1\right) \\
P_{d}(i)=\operatorname{proba}\left\{d_{k, l}=j\right\} ;\left(j=-N_{g}+1, . ., N_{g}-1\right)
\end{array}\right.
$$

Nous présentons les huit premiers attributs extraits à partir des H.S.D, qui sont les plus utilisés et les plus pertinents en termes de description de texture (Unser, 1986). Moyenne (Moy) : C'est la moyenne des niveaux de gris.

$$
\text { May }=\frac{1}{2} \sum_{i} i \cdot \hat{P}_{s}(i)=\mu .
$$

Variance (Var) : Cet attribut calcule la propagation autour de la moyenne.

$$
\operatorname{Var}=\frac{1}{2} \sum_{i}\left((i-2 \mu)^{2} \cdot \hat{P}_{s}(i)+\sum_{j}\left(j^{2} \cdot \hat{P}_{d}(j)\right) .\right.
$$

Probabilité maximale (Pmax) : La valeur maximale des fréquences d'apparition des couples de niveaux de gris.

$$
\operatorname{Pmax}=\max \left\{\hat{P}_{s}(i)\right\} .
$$

Énergie (Eng) : Cet attribut permet de quantifier l'homogénéité globale de la texture. En effet, l'énergie a une valeur faible s'il existe peu de transitions de niveaux de gris.

$$
\text { Eng }=\sum_{i} \hat{P}_{s}(i)^{2} \cdot \sum_{j} \hat{P}_{d}(j)^{2}
$$




\begin{tabular}{|c|c|c|c|}
\hline Critères $\quad$ Méthodes & Modèles de Markov/ Modèle autorégressif & Motifs Ternaires Locaux & Modèles multifractals \\
\hline Utilisation & Limitée & Limitée & Étendue \\
\hline $\begin{array}{l}\text { Nombre } \quad \text { de } \\
\text { paramètres à définir }\end{array}$ & Faible & Elevé & Faible \\
\hline Temps de calcul & Elevé & Faible & Faible \\
\hline Multi-résolution & Non & Non & Oui \\
\hline
\end{tabular}

TABLE 3 : Synthèse des comparaisons des méthodes d'analyse basée modèle.

Contraste (Cont) : Il a un comportement inverse de l'homogénéité locale.

$$
\text { Cont }=\sum_{j} j^{2} \cdot \hat{P}_{d}(j)
$$

Corrélation (Corr) : Cet attribut quantifie l'uniformité des distributions des niveaux de gris; il mesure la dépendance linéaire des niveaux de gris. Des valeurs de corrélation élevées indiquent un certain ordre local des niveaux de gris.

$$
\text { Corr }=\frac{1}{2}\left(\sum_{i}(i-2 \mu) \cdot \hat{P}_{s}(i)-\sum_{j} j^{2} \cdot \hat{P}_{d}(j)\right) .
$$

Entropie (Ent) : C'est un indicateur du désordre (caractère aléatoire) de la texture. L'entropie permet aussi de caractériser le degré de granularité de l'image.

$$
\text { Ent }=\begin{aligned}
& -\sum_{i}\left(\hat{P}_{s}(i) \cdot \log \left(\hat{P}_{s}(i)\right)\right) \\
& -\sum_{j}\left(\hat{P}_{d}(j) \cdot \log \left(\hat{P}_{d}(j)\right)\right)
\end{aligned}
$$

Homogénéité (locale) (Hom) : Plus cet attribut est élevé plus la texture possède des régions homogènes dans une direction $\theta$.

$$
\text { Hom }=\sum_{j}\left(\frac{1}{1+j^{2}} \cdot \hat{P}_{d}(j)\right),
$$

avec $\hat{P}_{s}(i)$, l'élément à l'indice $i$ de l'histogramme de sommes normalisé et $\hat{P}_{d}(j)$, l'élément à l'indice $j$ de l'histogramme de différences normalisé.

\subsection{Méthode d'analyse multifractale}

À noter qu'il existe deux méthodes basées sur l'approche multifractale (Tahiri Alaoui, 2006) :

- La première méthode donne l'information locale (représentée par "l'exposant de singularité"). Ses attributs de texture sont des statistiques sur l'image des singularités qui décrit la présence des différentes irrégularités.

- La deuxième méthode fournit l'information globale et locale (donnée par "le spectre multifractal"). Des statistiques sur ce spectre peuvent être considérées comme des attributs de texture.

L'analyse par la deuxième méthode utilise plusieurs dimensions fractales, plutôt qu'une seule dimension. Par ailleurs, la première méthode est préférable dans les applications nécessitant une information locale.

Puisque notre but est d'extraire l'information statistique locale, nous utilisons la première méthode.

Et parmi les techniques d'analyse multifractale, nous utilisons celle de Turiel (Turiel and Parga, 2000a,b) puisqu'elle admet des avantages par rapport aux techniques classiques grâce à l'intervention des ondelettes. En fait, nous n'avons considéré, de l'algorithme de Jacopo Grazzini (2003), que la section des calculs d'exposant : La construction d'une mesure multifractale, puis le choix d'une ondelette analysante suivie par une projection en ondelette de la mesure et finalement l'estimation des exposants qui sont illustrés par l'équation 13 suivante.

$$
\alpha(\vec{x})=\lim _{r \rightarrow 0} \frac{\log T_{\Psi}^{r} \mu(\vec{x})}{\log r} .
$$

Avec $\alpha$ l'exposant de singularité, $\mu$ la mesure multifractale, $\Psi$ l'ondelette, $\vec{x}$ un point de l'image et $r$ un facteur d'échelle de traitement (le facteur de dilatation) et $T_{\Psi}^{r} \mu$ une projection en ondelette. Notons que dans (Turiel and Praga, 2000), les auteurs ont défini la classe des fonctions d'ondelette adaptées à l'analyse des singularités (pour lesquelles la relation précédente est vérifiée).

Pour notre approche d'identification de signature combinée, nous choisissons l'ondelette lorentzienne (avec l'ordre $\gamma=1$ ) pour estimer les exposants de singularité. Son expression générale est donnée par l'équation 14 :

$$
\Psi(\vec{x})=\left(1+|\vec{x}|^{2}\right)^{-\gamma} .
$$

\section{Mesures et attributs utilisés dans le processus proposé}

La deuxième étape de notre approche d'identification de signature sert à déterminer les mesures de texture pour la signature puis à déterminer les attributs de texture utilisés ultérieurement dans un processus proposé. Notre idée consiste alors à ne pas utiliser toutes les méthodes d'analyse de texture les plus adéquates pour notre cas. Nous allons utiliser uniquement les méthodes qui génèrent des attributs quantifiant certaines mesures qui nous semblent nécessaires pour caractériser notre type de texture, au lieu d'effectuer des tests sur tous les attributs possibles. 


\subsection{Mesures de texture pour notre signature}

Les descripteurs de texture (appelés aussi attributs de texture) quantifient des propriétés de texture (appelées aussi mesures de texture) telles que la régularité, la rugosité, la directionnalité, la linéarité, la finesse. Law (1980) est l'un des chercheurs qui ont identifié les mesures qui jouent un rôle important dans la description de texture : l'uniformité, la densité, la finesse, la rugosité, la régularité, la linéarité, la direction, la phase et la fréquence. Certaines de ces qualités sont dépendantes. Par exemple, la fréquence n'est pas indépendante de la densité. D'autre part, la propriété de direction ne s'applique qu'aux textures directionnelles.

Les mesures jouant un rôle important dans la description de texture pour notre cas sont : l'uniformité, la finesse, la rugosité et la régularité.

\subsection{Attributs utilisés dans le processus proposé}

L'approche qui consiste à extraire puis à combiner les descripteurs de couleur et les descripteurs de texture, est un bon moyen pour obtenir des résultats visuels et numériques satisfaisants. Pour des raisons de simplicité, nous utilisons uniquement la bande spectrale proche infrarouge (PIR) de notre image satellitaire qui est la bande la plus privilégiée dans notre cas pour extraire les descripteurs des espèces forestières, puisqu'elle est sensible aux radiations des zones forestières.

En raisonnant par analogie, l'approche qui consiste à extraire puis à combiner les descripteurs d'intensité lumineuse, à partir de la bande PIR, et les descripteurs de texture, nous semble une bonne approche. Dans ce contexte, les attributs de texture et les attributs d'intensité lumineuse seront regroupés pour former notre signature. Dans la littérature nous avons été confrontés à un grand nombre d'attributs d'intensité lumineuse. En général, ces attributs expriment les moments statistiques. Dans notre cas, nous utiliserons les attributs: "moyenne" et "variance".

Concernant les attributs de texture, ils vont être choisis en tenant compte d'une part des mesures choisies et d'autre part des trois méthodes d'analyse de texture les plus adéquates (cf. Section 2).

Tout d'abord, nous nous intéressons aux attributs extraits de la méthode d'analyse des histogrammes de sommes et de différences.

D'après Clausi (2002), les six attributs d'Unser les plus utilisés sont corrélés entre eux. En fait, il a établit trois catégories d'attributs de texture fondées sur leur intercorrélation :

- Les attributs "contraste" et "homogénéité" quantifient la mesure de rugosité de texture;

- Les attributs "énergie", "entropie" et "probabilité maximale" quantifient la finesse de texture;

- L'attribut "corrélation" quantifie l'uniformité.

Clausi (2002) proscrit alors l'emploi simultané de plusieurs attributs de texture appartenant au même groupe, dans un processus de classification texturale.
Alors, nous pouvons dire qu'en utilisant uniquement cette méthode d'analyse, la texture sera caractérisée par trois mesures de texture (rugosité, finesse et uniformité) parmi les quatre mesures choisies.

Concernant la mesure non encore considérée (la régularité), nous utilisons la méthode d'analyse multifractale via la transformée en ondelettes générant des exposants de singularité (exposants de Hölder).

À noter que l'exposant de Hölder nous permet de caractériser la structure multifractale de l'image, comme il permet de caractériser "parfaitement" la rugosité de texture des images.

Plus précisément cet exposant quantifie la rugosité en changement d'échelle (Krim and Indekeu, 1993). Ainsi, nous proposons d'utiliser l'exposant de Hölder à la place de l'attribut de rugosité extrait de la méthode des H.S.D puisque cet exposant permet de caractériser la rugosité de texture avec l'avantage d'analyse multi-échelle.

\subsection{Organigramme du processus}

La troisième étape de notre approche d'identification de signature consiste à appliquer un processus d'identification de signature combinée proposé. Les paramètres de notre processus sont les paramètres des deux méthodes d'analyse utilisées, qui sont la taille de fenêtre d'analyse $f$ définissant le voisinage d'un pixel (paramètre de la méthode multifractale et la méthode des H.S.D), le pas d'échantillonnage $p$ et la direction d'échantillonnage $d$ (paramètres de la méthode des H.S.D).

\section{Processus d'identification de signature combinée}

Le paramètre $d$ ne va pas être une variable d'entrée du processus car il est fixé par une valeur appropriée à notre donnée. Alors, ce processus utilise uniquement des valeurs de couple de paramètres $(f, p)$.

Un sous-processus est effectué pour chaque couple de paramètres $(f, p)$ et générant comme valeurs de retour deux taux de classification.

Chaque taux est une mesure d'évaluation de la classification d'une image de test par une signature candidate différente.

Et un choix du taux maximal parmi tous les taux obtenus, permet de déterminer tous les paramètres optimaux du processus et les attributs de la signature :

$$
(f, p, s i g)_{o p t}=\underset{(f, p)}{\arg \max }\left(\operatorname{Taux}_{(f, p)}\right) .
$$

L'organigramme de ce processus est présenté dans la Figure 2.

\subsection{Sous-processus pour un couple de paramètres}

Le sous processus d'identification de signature combinée (cf. Figure 3) génère, pour une combinaison des 


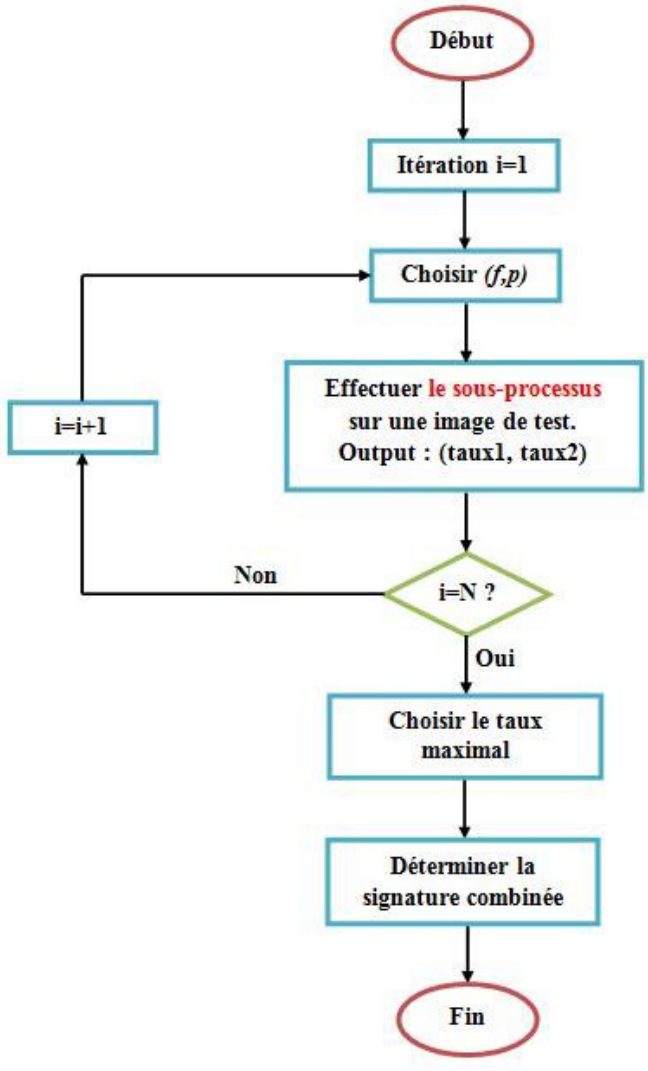

FIGURE 2 : Organigramme du processus proposé d'identification de signature combinée. paramètres $f$ et $p$, deux taux de classification (appelés $\operatorname{Taux} 1_{(f, p)}$ et $\left.\operatorname{Taux} 2_{(f, p)}\right)$ en utilisant deux signatures qui se diffèrent uniquement au niveau des attributs d'intensité lumineuse.

Concernant les attributs de texture de la signature, chaque mesure est quantifiée par un seul attribut sauf la mesure de finesse qui peut être quantifiée par plus qu'un attribut candidat. Pour cela, nous proposons d'effectuer un processus de choix d'attribut de finesse.

Ce processus de choix consiste à évaluer la classification des différentes images des attributs de texture quantifiant la finesse. Ainsi, les résultats dépendent du couple $(f, p)$. Pour cette raison, nous proposons d'effectuer le processus de choix pour chaque couple de paramètres $(f, p)$. Cela justifie la proposition d'inclure ce processus de choix dans le sous-processus d'identification de signature combinée. Alors, pour un couple de paramètres, l'attribut de finesse est le même pour les deux signatures candidates, mais n'est pas nécessairement le même pour tous les signatures quelque soit $(f, p)$. Les valeurs des variables d'entrée $f$ et $p$ vont être utilisés dans la méthode des H.S.D qui nous donne deux attributs de texture : un attribut mesurant l'uniformité et un autre mesurant la finesse.

L'attribut de finesse est déterminé à l'aide d'un processus de choix appliqué sur ce que nous appelons un "fichier de finesse". Ce fichier est formé par trois images des attributs de finesse : "énergie", "entropie" et "probabilité maximale".

De plus, la valeur du paramètre $f$ sera utilisée aussi dans la méthode multifractale engendrant l'image des exposants de Hölder qui nous permet de quantifier la régularité et la rugosité de texture. L'attribut extrait de cette méthode n'est rien d'autre qu'une statistique sur l'image des exposants. Dans notre cas, c'est la statistique "moyenne".

En outre, pour former la signature, nous extrairons aussi, comme attributs d'intensité lumineuse, des statistiques calculées sur une fenêtre glissante de taille $f$. En fait, nous utiliserons deux attributs d'intensité qui sont la "moyenne" et la "variance", afin de choisir l'un des deux, ou tous les deux, dans la composition de la signature.

Alors, notre sous-processus permet de tester, pour une combinaison des paramètres $(f, p)$, la capacité de deux signatures à classifier notre image.

Pour cette raison, nous créons pour chaque $(f, p)$ deux fichiers candidats (appelés Fichier 1 et Fichier 2 ) tel que chacun contient des images des attributs constituant une signature candidate.

\subsection{Choix des valeurs possibles des paramètres du processus}

La plupart des chercheurs testent un grand nombre de combinaisons possibles du triplet $(f, d, p)$ afin de choisir la combinaison optimale. Nous essayons de corriger ce point faible afin d'avoir une procédure simple et fiable et de complexité temporelle réduite. En fait, notre processus d'identification de signature nécessite 


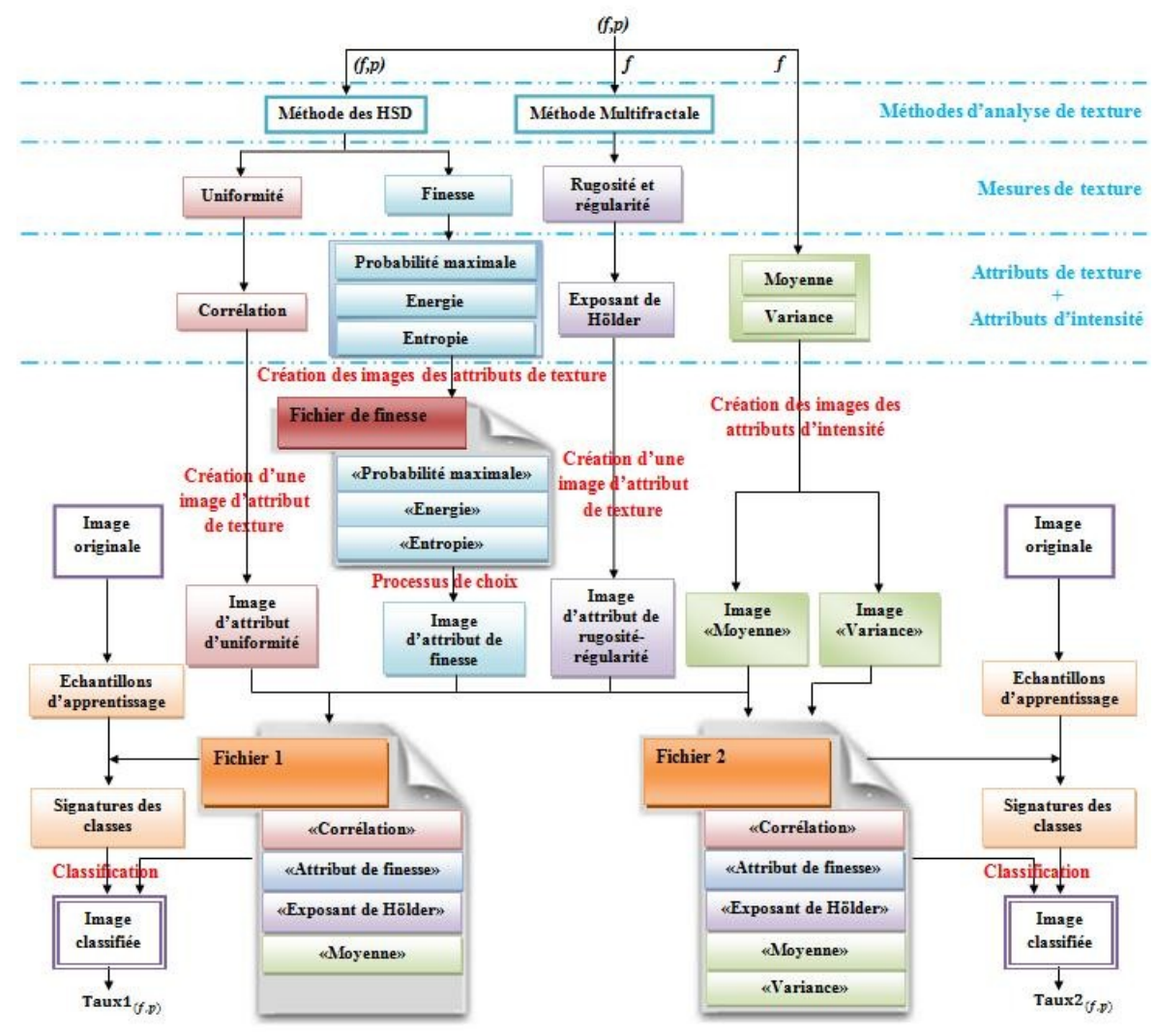

FIGURE 3 : Schéma du sous-processus d'identification de signature combinée pour un couple de paramètres $(f, p)$.

un petit nombre de valeurs possibles (les plus utiles) des paramètres, et sert à déterminer les valeurs optimales.

Pour notre cas, la taille de la fenêtre d'échantillonnage est forcément supérieure ou égale au diamètre d'une couronne d'arbre forestier, pour bien capter la texture d'une espèce forestière. Mais, les arbres forestiers existants dans une image satellitaire de scène forestière n'ont pas forcément la même taille.

Un nombre trop supérieur au diamètre de l'arbre ayant la plus grande taille dans l'image toute entière ne peut pas être une valeur possible de $f$. En effet, la caractérisation des arbres ayant des tailles trop inférieures à la taille choisie va être erronée, car il y a un grand risque que la fenêtre couvre des espèces forestières différentes.

En conséquence, les valeurs possibles de $f$ appartiennent à l'intervalle des tailles des arbres existants dans l'image. Comme nous pouvons avoir des valeurs qui sont un peu plus grandes que la borne supérieure de cet intervalle. Cependant, le choix des valeurs les plus utiles de cet intervalle reste difficile.

En effet, l'utilisation d'une valeur de $f$ proche du diamètre de l'arbre ayant la plus petite taille dans l'image (la limite inférieure de l'intervalle), peut causer des problèmes pour la caractérisation des arbres de grand diamètre.

Ces problèmes sont dus au fait que l'information de texture, qui caractérise ce type d'arbre est insuffisante et imprécise. De même, l'utilisation d'une taille proche du diamètre de l'arbre ayant la plus grande taille dans l'im- age, peut engendrer des problèmes pour la caractérisation de très petits arbres.

Alors, pour obtenir l'ensemble de valeurs de $f$ les plus utiles pour notre processus et pour une image particulière, nous faisons des tests en fixant tous les paramètres et ne variant que le paramètre $f$ dans l'intervalle choisi.

En ce qui concerne le paramètre de pas d'échantillonnage $p$, Bugnet et al. (2003) par exemple ont déduit qu'il est préférable de prendre les valeurs de $p$, indépendamment de la taille de fenêtre $f$. En d'autres termes, les combinaisons avec un grand pas $p$ doivent être évitées même si $f$ a une valeur élevée, afin d'augmenter la capacité de capturer de la micro-texture.

Concernant la direction d'échantillonnage $d$, la moyenne de toutes les directions est le meilleur choix.

En fait, plusieurs chercheurs ont assumé l'isotropie des différentes textures et ainsi ont utilisé comme canaux d'entrée à leur algorithme de classification, les images des moyennes des attributs de texture calculées pour les quatre directions d'échantillonnage.

Cependant, il est démontré que dans le cas d'images à haute résolution, les zones ombragées créées par les arbres induisent une anisotropie de la texture, en particulier dans une direction proche de la direction de l'illumination du soleil (Saint-Onge, 1994). Ainsi, dans notre cas, $d$ est fixé au début, au lieu de faire intervenir ce paramètre dans les tests, dans le but de développer une procédure 
simple et fiable. Le paramètre $d$ va prendre la valeur indiquant la direction d'illumination du soleil.

\subsection{Processus de choix de l'attribut de finesse de texture}

Bugnet et al. (2003) ont utilisé une méthodologie pour extraire une signature à partir de la méthode des H.S.D. Cette méthodologie est adaptée afin d'obtenir un outil pour le choix de l'attribut de finesse de texture.

Notre processus de choix est effectué pour chaque couple de paramètres $(f, p)$. Il se résume essentiellement en deux étapes: Une création des images des attributs de finesse, suivie par un choix de l'attribut de finesse optimal.

Une image d'un attribut est construite par les valeurs de cet attribut, en utilisant la technique de fenêtre glissante parcourant toute l'image. Cette technique est utilisée aussi pour créer l'image d'attribut d'intensité.

La première étape du processus de choix consiste alors en la création du "fichier de finesse" pour un couple $(f, p)$.

La deuxième étape consiste à faire, en premier lieu, un classement préliminaire des différents attributs et ceci en évaluant la capacité de chaque attribut à dégager les détails et à rehausser les discontinuités au sein des différentes classes. C'est une analyse visuelle des images du fichier de finesse. Et en deuxième lieu, une analyse numérique des résultats de classification des différentes images, par le calcul des matrices de confusion et l'évaluation de leurs mesures, est effectuée.

\subsection{Classification}

Notre processus d'identification de signature s'appuie sur une classification supervisée. Pour des raisons de simplicité nous choisissons d'utiliser la technique de classification supervisée k-means (Macqueen, 1967) avec un critère de distance euclidienne minimale. En fait, nous minimisons la distance euclidienne entre un pixel (caractérisé par son vecteur d'attributs) et le centre de chaque classe (caractérisé par le vecteur moyenne) calculé à partir des échantillons d'apprentissage. Le pixel est assigné à la classe associée à la distance minimale. II est à noter que notre classification est basée sur la similitude des propriétés texturales.

Puisque le processus d'identification de signature combinée proposé crée des images des attributs formant notre signature, la classification d'une image est faite par un algorithme appliqué sur ces images. Autrement dit, l'image à classifier est une superposition des images qui correspondent aux attributs de signature (attributs de texture et attributs d'intensité). C'est-à-dire, chaque pixel de l'image à classifier est un vecteur des valeurs des attributs.

\section{Identification du poids pour la signature combinée}

\subsection{Besoin de pondérer la signature}

Le cas qui nous a fait penser à pondérer la signature, est le cas de classification d'un pixel avec une signature (notée $S$ ) ayant plusieurs attributs tel que chaque attribut donne une décision de classification différente, et la décision par la signature $S$ est celle de la classification par une signature formée uniquement par un attribut particulier (noté $X$ ). Si nous supposons que l'attribut $X$ a un certain taux d'erreur de décision élevé (c'est-à-dire son degré de confiance dans la décision est presque nul), alors la décision de classification par la signature $S$ peut changer d'une classe à une autre.

Autrement dit, c'est le cas de classification avec la supposition d'avoir un attribut de la signature ayant un taux d'erreur élevé qui influence sur la décision de classification.

En fait, la classification sans tenir en compte les taux d'erreur de différents attributs, peut être erronée. Mais, est-ce que l'attribut de signature a vraiment un certain taux d'erreur? Pour répondre à cette question, nous considérons deux textures différentes (cf. Figure 4) ayant le même degré d'une mesure de texture. Dans ce cas c'est la dimension fractale. La classification du pixel central de deux fenêtres de texture (cf. Figures $4 a$ et b) par une signature formée uniquement par un attribut de texture mesurant la dimension fractale, donne deux décisions de classification identiques. Donc cet attribut ne peut pas identifier la texture. Nous pouvons dire alors que cet attribut possède un taux d'erreur d'identification de texture très élevé.

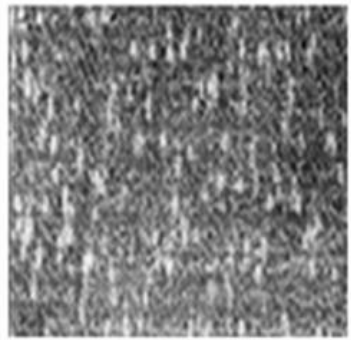

(a)

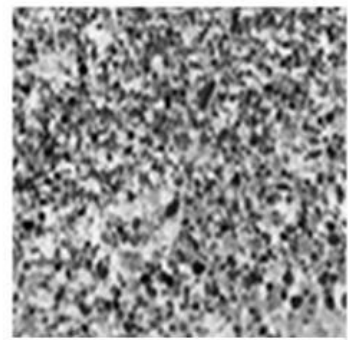

(b)
FIGURE 4 : Deux textures différentes ayant la même valeur d'un attribut de texture (Xia et al., 2010) : (a) texture $T 1$, (b) texture $T 2$.

Pour généraliser, nous pouvons dire que chaque attribut de signature a un taux d'erreur qui peut influencer la décision de classification. Alors, la réponse largement acceptée à la question posée précédemment est affirmative.

Alors notre amélioration de résultat de classification consiste à accorder une faible importance (influence) dans la décision de classification à l'attribut ayant un taux d'erreur élevé et vice versa. En fait, notre idée consiste à attribuer un certain poids à chaque attribut de notre signa- 


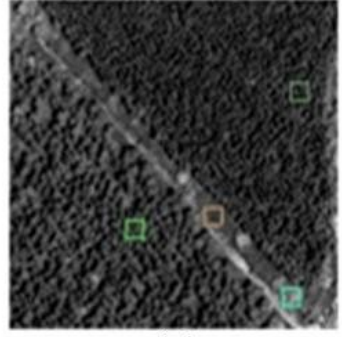

(a)

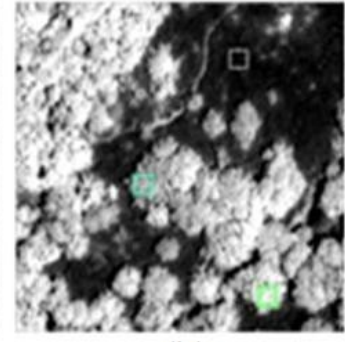

(b)

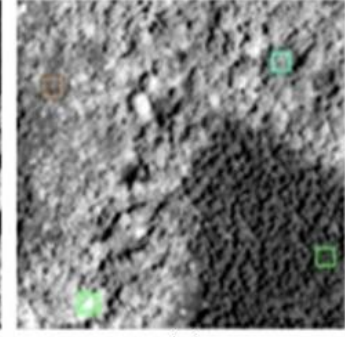

(c)

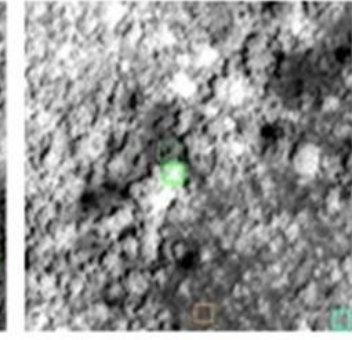

(d)

FIGURE 5 : Des extraits de l'image initiale : (a) image A, (b) image B, (c) image C, (d) image D.

ture, indiquant son importance dans la décision de classification.

L'avantage d'utiliser la distance Euclidienne, pour prendre la décision de classification, réside dans la simplicité et la complexité réduite. Cependant, la formule de cette distance accorde la même importance aux différents attributs de vecteur.

Pour cette raison, notre idée consiste à intégrer les poids dans la formule de distance afin d'exprimer l'importance de chaque attribut de signature dans le calcul de distance (un attribut est un élément important dans le calcul de distance si son poids est grand).

Notre idée est inspirée de Giap Nguyen qui a utilisé une formule modifiée de la distance Euclidienne afin de calculer la distance entre deux traits et ceci en ajoutant un poids pour chaque caractéristique de trait. Alors, la formule de distance utilisée dans l'algorithme de classification va être définie comme suit :

$$
D_{p}(C)=\sqrt{\sum_{k=1}^{n_{p}} p_{k}\left(v_{p}-v_{r}(C)\right)^{2}},
$$

avec $C$ une classe, $v_{p}$ le vecteur caractéristique du pixel $p, n_{p}$ le nombre des attributs constituant la signature, $v_{r}(C)$ la signature de classe $C$ et $p_{k}$ le poids correspondant à l'attribut $k$.

\subsection{Identification du poids}

Le poids d'un attribut et son taux d'erreur dans la classification sont dépendants et plus précisément complémentaires. De plus, notre poids varie entre 0 et 1 , alors : Poids (attribut) = 1- taux d'erreur (attribut).

Par ailleurs, puisque la signature peut être composée de plusieurs attributs, alors le poids d'un attribut peut être défini comme étant une mesure d'évaluation de la bonne classification utilisant uniquement cet attribut comme signature. Et parmi les mesures d'évaluation, nous choisissons la mesure de la précision globale notée $P G$ (en anglais overall accuracy) car théoriquement elle satisfait nos besoins :

$$
P G=\frac{\text { nombre de pixels correctement classifiés }}{\text { nombre total de pixels }}
$$

En littérature, le choix des attributs de signature est fortement conditionné par la nature de l'image et les classes. Par contre, notre signature surmonte cette lacune, puisque elle peut être utile pour tout type d'image satellitaire de scène forestière et ceci grâce à l'utilisation des poids.

\section{Expérimentation}

Cette section illustre l'application de notre approche sur une image satellite (QuickBird) à haute résolution $(0,6 \mathrm{~m})$ d'une zone au nord de la Tunisie (Ain Drahim). L'inventaire forestier correspondant à la même scène de l'image souligne six classes : SPC : Sol Peu Couvert, SN : Sol Nu, CL : Chêne Liège, CZ : Chêne Zen, PP : Pin Pignon, PM : Pin Maritime.

Quatre extraits (sous-images) de l'image originale, comprenant des zones représentatives des espèces forestières, sont choisis à des fins d'expérimentation. Ces sous-images sont notées $A, B, C$ et D. Elles sont choisies de telle façon que toutes les classes présentes dans l'image originale existent dans l'ensemble de ces sous-images. À noter que la visualisation des résultats d'expérimentation sur des extraits de petites dimensions est mieux que celle du résultat expérimental sur l'image entière. En effet, les extraits nous permettent de distinguer visuellement les différentes espèces, et donc l'analyse visuelle sera plus facile. La Figure 5 présente ces extraits indiquant la disposition de quelques échantillons d'apprentissage, et la Figure 6 présente les images inventaires correspondantes.

Par ailleurs, nous pouvons dire que l'information fournie par notre image inventaire est imprécise, car plusieurs polygones correspondent à des mélanges d'espèces différentes. En fait, l'inventaire apporte souvent une information globale sur l'espèce dominante et informe souvent sur des mélanges d'espèces.

De plus l'information fournie est incertaine puisque l'image inventaire et l'image satellitaire dont nous disposons n'ont pas un même référentiel temporel (la plus ancienne est l'image inventaire). Donc l'information donnée par l'image inventaire n'est pas totalement conforme à la vérité.

Pour cette raison, le choix des échantillons d'apprentissage et des échantillons de test se fait en se basant essentiellement sur l'image originale et à l'aide de l'image 


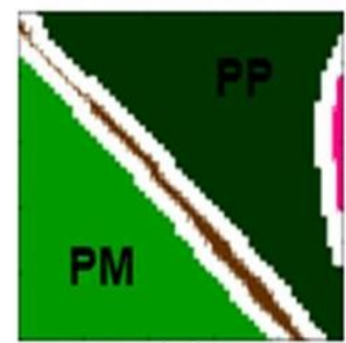

(a)

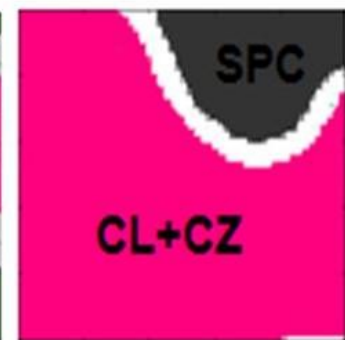

(b)

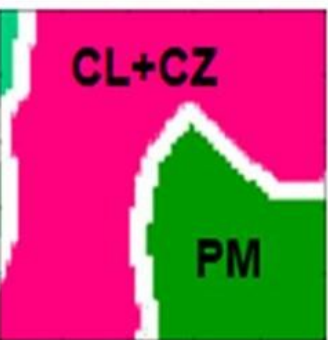

(c)

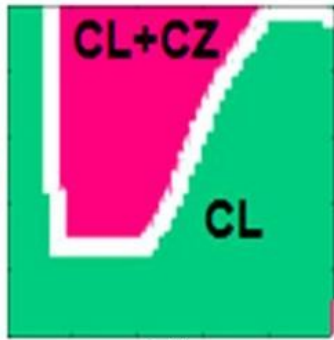

(d)

FIGURE 6 : Images inventaires correspondantes : (a) inventaire de A, (b) inventaire de B, (c) inventaire de C, (d) inventaire de D.

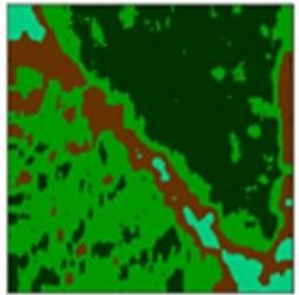

(a)

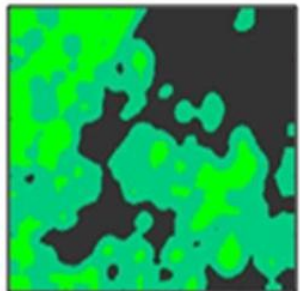

(b)

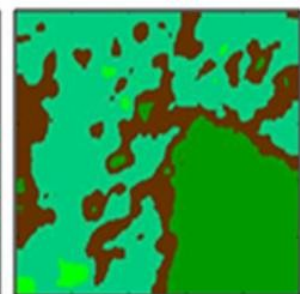

(c)

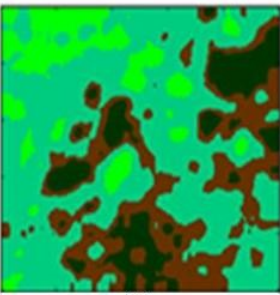

(d)

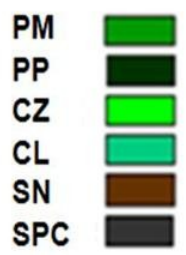

(e)

FIGURE 7 : Résultats de classification : (a) classification de A, (b) classification de B, (c) classification de C, (d) classification de D, (e) légende.

\begin{tabular}{|c|c|c|c|c|c|}
\hline & SN & CL & PP & PM & EC \\
\hline SN (Sol Nu) & 68,83 & 22,46 & 0,00 & 8,69 & 0,50 \\
\hline CL (Chêne Liège) & 37,70 & 62,29 & 0,00 & 0,00 & 0,61 \\
\hline PP (Pin Pignon) & 0,89 & 0,02 & 79,14 & 19,93 & 0,14 \\
\hline PM (Pin Maritime) & 10,80 & 0,00 & 14,49 & 74,69 & 0,25 \\
\hline EO (Erreur d'Omission) & 0,31 & 0,38 & 0,21 & 0,25 & \\
\hline
\end{tabular}

TABLE 4 : Matrice de confusion de la classification de l'image A.

\begin{tabular}{|c|c|c|c|c|}
\hline & SPC & CL & CZ & EC \\
\hline SPC (Sol Peu Couvert) & 97,14 & 2,85 & 0,00 & 0,00 \\
\hline CL (Chêne Liège) & 1,17 & 95,09 & 3,72 & 0,17 \\
\hline CZ (Chêne Zen) & 0,00 & 13,19 & 86,80 & 0,03 \\
\hline EO (Erreur d'Omission) & 0,02 & 0,04 & 0,13 & \\
\hline
\end{tabular}

TABLE 5 : Matrice de confusion de la classification de l'image B.

\begin{tabular}{|c|c|c|c|c|c|}
\hline & SN & CL & CZ & PM & EC \\
\hline SN (Sol Nu) & 72,77 & 22,39 & 00,00 & 04,84 & 0,78 \\
\hline CL (Chêne Liège) & 14,20 & 85,33 & 00,46 & 00,00 & 0,03 \\
\hline CZ (Chêne Zen) & 00,36 & 32,68 & 66,96 & 00,00 & 0,15 \\
\hline PM (Pin Maritime) & 03,18 & 00,00 & 00,00 & 96,82 & 0,00 \\
\hline EO (Erreur d'Omission) & 0,27 & 0,15 & 0,33 & 0,03 & \\
\hline
\end{tabular}

TABLE 6 : Matrice de confusion de la classification de l'image C.

\begin{tabular}{|c|c|c|c|c|c|}
\hline & SN & CL & CZ & PP & EC \\
\hline SN (Sol Nu) & 71,45 & 08,61 & 0,00 & 19,93 & 0,50 \\
\hline CL (Chêne Liège) & 11,83 & 88,05 & 0 & 0,12 & 0,07 \\
\hline CZ (Chêne Zen) & 0,00 & 11,37 & 88,63 & 0,00 & 0,00 \\
\hline PP (Pin Pignon) & 12,36 & 0,00 & 0,00 & 87,64 & 0,13 \\
\hline EO (Erreur d'Omission) & 0,28 & 0,12 & 0,11 & 0,12 & \\
\hline
\end{tabular}

TABLE 7 : Matrice de confusion de la classification de l'image D. 


\begin{tabular}{|c|c|c|c|c|}
\hline & Image A & Image B & Image C & Image D \\
\hline Kappa & 60,53 & 90,43 & 81,05 & 79,41 \\
\hline PG & 76,11 & 94,01 & 89,03 & 86,24 \\
\hline
\end{tabular}

TABLE 8 : Indices de kappa (en \%) et mesures de la précision globale (en \%) de la classification des images A, B, C et D.

inventaire correspondante.

Et malgré que la carte de référence et le résultat de la classification n'aient pas la même nature, l'image inventaire reste une base pour l'interprétation visuelle des résultats de classification dans la mesure où une bonne décision de classification devra correspondre à l'une des espèces codées par le polygone correspondant de l'inventaire.

Nous ajoutons une règle d'équivalence, concernant l'ajout des poids, qui améliore significativement les résultats pour notre image. Cette règle consiste à annuler l'effet des attributs (Poids $\leftarrow 0$ ) ayant des poids faibles dans la décision de classification (Poids $<0,5$ ). Cette règle consiste à annuler l'effet de certains attributs, ayant des poids faibles, dans la décision de classification. Il est à noter que cette règle améliore significativement les résultats pour notre image.

Les résultats de classification par notre signature combinée pondérée des extraits (image $A$ - image D) sont présentés dans la Figure 7.

Une interprétation visuelle des résultats de classification montre que notre objectif est atteint.

En effet, nous avons pu identifier et départager les différentes espèces forestières existantes dans chaque sous-image.

En plus, nous avons pu identifier les limites des zones denses des espèces forestières et les arbres dispersés. Les limites entre ces zones sont très bien identifiées. Et bien que généralement l'identification exacte des formes, seulement avec l'information de texture, soit très difficile, les formes des parcelles sont bien claires.

Une interprétation numérique par le calcul des différentes matrices de confusion (Tableau 4 - Tableau 8) et leurs statistiques (les erreurs de commission EC, les erreurs d'omission EO, les indices de kappa et les mesures de précision globale $P G$ ) confirme les résultats visuels. Le Tableau des statistiques de performance de classification des différents extraits (image $A$ - image $D$ ) indique que les valeurs de kappa et de PG sont satisfaisantes (kappa entre $60,53 \%$ et $90,43 \%$ et PG entre $76,11 \%$ et 94,01\%).

Plus particulièrement, par exemple la classification de l'image A nous permet de mesurer le pouvoir de notre signature de distinguer deux classes différentes (Pin Maritime et Pin Pignon) ayant un chevauchement radiométrique. Et malgré la difficulté de départager les deux espèces de pin, la signature a réussi d'affecter ces deux classes. Ce qui est expliqué aussi par les pourcentages élevés des pixels correctement classifiés, au diagonal de la matrice de confusion correspondante donnée par le Tableau 4.
Pour enrichir cette partie expérimentale, nous évaluons la classification des seize autres sous-images (Tableau 9). Nous constatons de ce Tableau la bonne qualité de classification expliquée par les mesures de précision globale élevées (entre $82,15 \%$ et $99,12 \%$ ), et des indices de kappa satisfaisants (entre $58,38 \%$ et $92,43 \%)$. En outre, l'analyse visuelle des images classifiées confirme ces résultats.

\section{Conclusion}

Nous avons présenté dans cet article une approche de détermination de signature de texture qui consiste à combiner un nombre de descripteurs en tenant compte des méthodes d'analyse de texture les plus adéquates pour notre contexte. La combinaison des attributs de texture n'est pas faite de façon aléatoire. En fait, nous avons utilisé les méthodes générant des attributs quantifiant certaines mesures qui nous semblent nécessaires pour caractériser notre type de texture. Ensuite, nous avons défini un poids pour chaque attribut de signature afin d'obtenir la signature combinée pondérée. L'application de l'approche proposée sur notre zone d'étude confirme l'efficacité de cette approche. Elle prouve l'apport de l'utilisation des exposants de Hölder avec certains attributs d'Unser et autres attributs d'intensité lumineuse dans l'amélioration de la qualité de classification. En plus, elle prouve l'apport de l'utilisation des poids pour chaque attribut de notre signature.

\section{Références}

Abadi M., 2008. Couleur et texture pour la représentation et la classification d'images satellite multi-résolutions. PhD thesis, University of the French West Indies and Guiana, France.

Akono A., Tonyé E., Rudant J.P., 2003. Classification texturale d'une image radar à synthèse d'ouverture dans une région volcanique : le cas de la région du Mont Cameroun. Remote Sensing, 3(2) :115-128.

Bugnet P., Cavayas F., Gagnon L., 2003. Vers la cartographie automatisée des surfaces boisées en milieu urbanisé fondée sur la texture d'images IKONOS panchromatiques : le cas de la Région Métropolitaine de Montréal. Canadian Journal of Remote Sensing, 29(6) :755-769. 

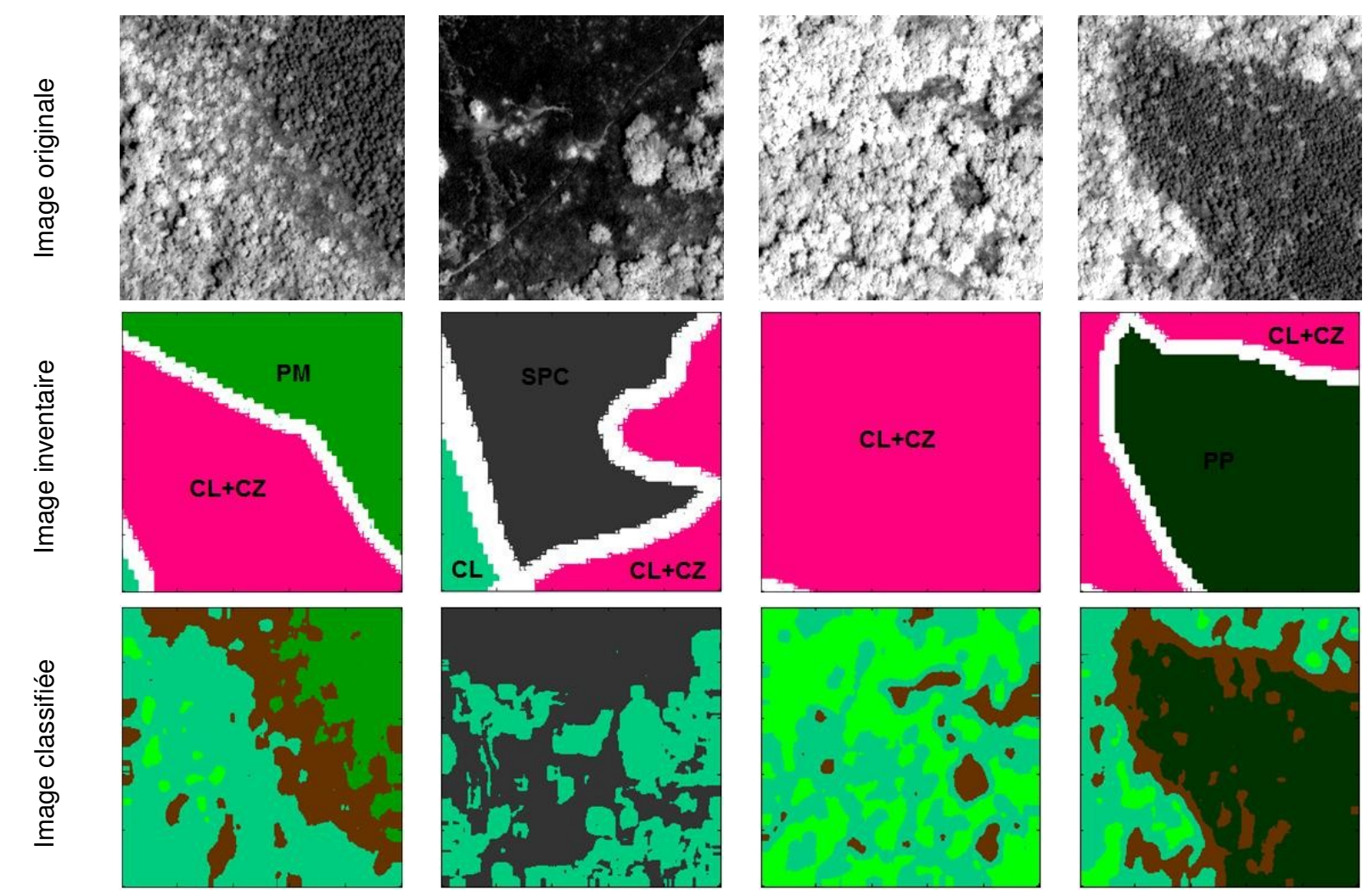

\begin{tabular}{cc} 
Kappa & 79,81 \\
PG & 86,81 \\
\hline
\end{tabular}
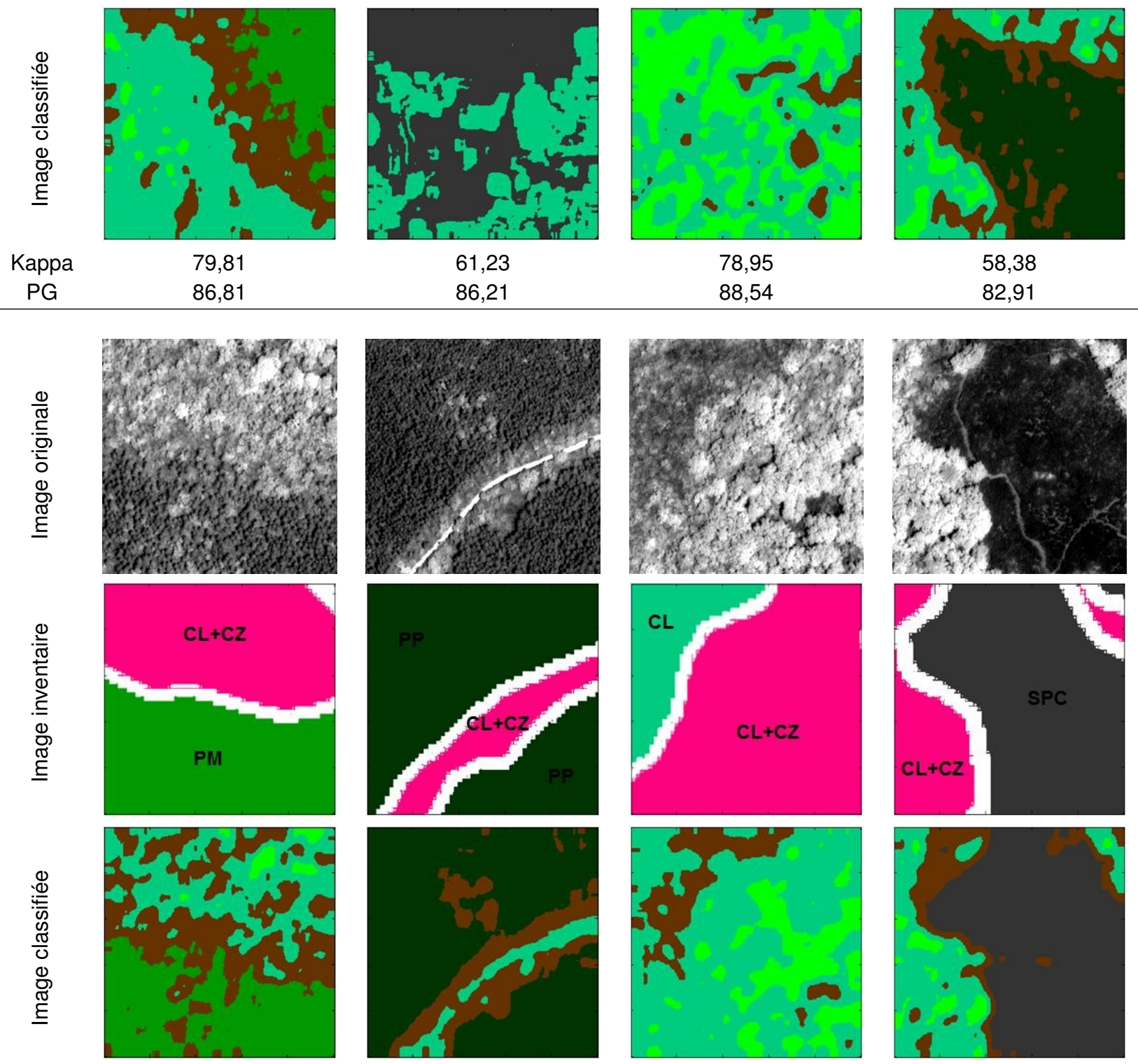

Kappa

72,71

74,62

86,33

58,38

82,91

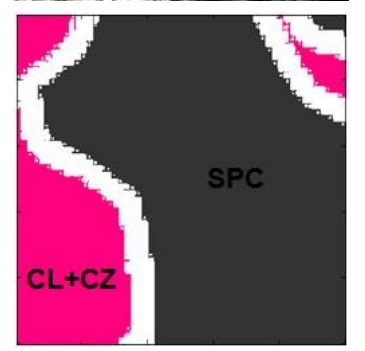

$P G$

82,47

96,26

91,42

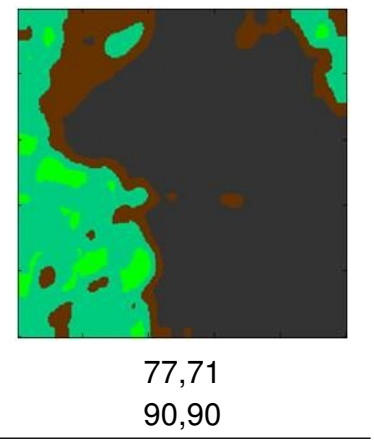



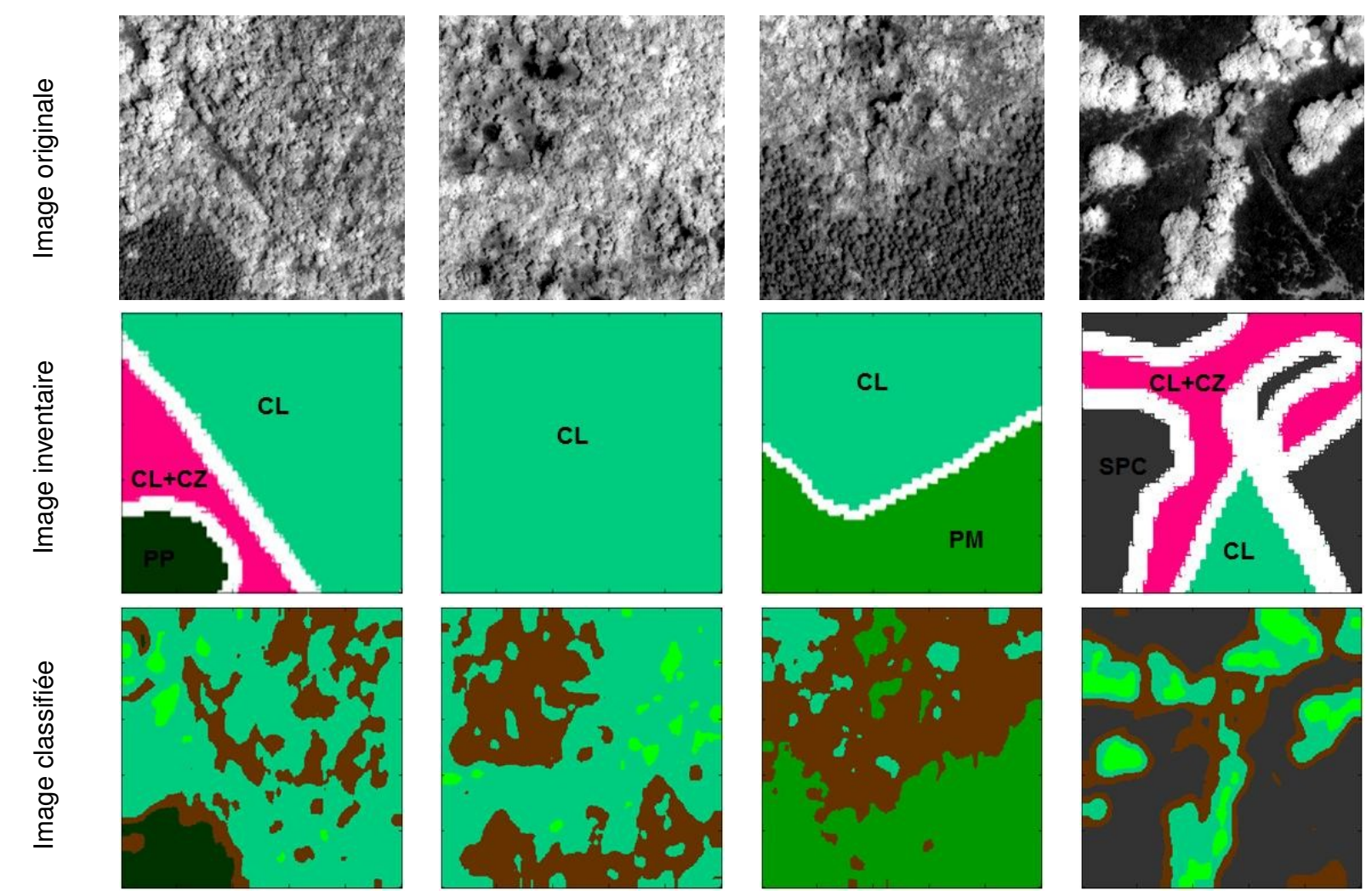

Kappa
PG

67,37

67,37

80,80

78,73

88,01

91,25

89,18
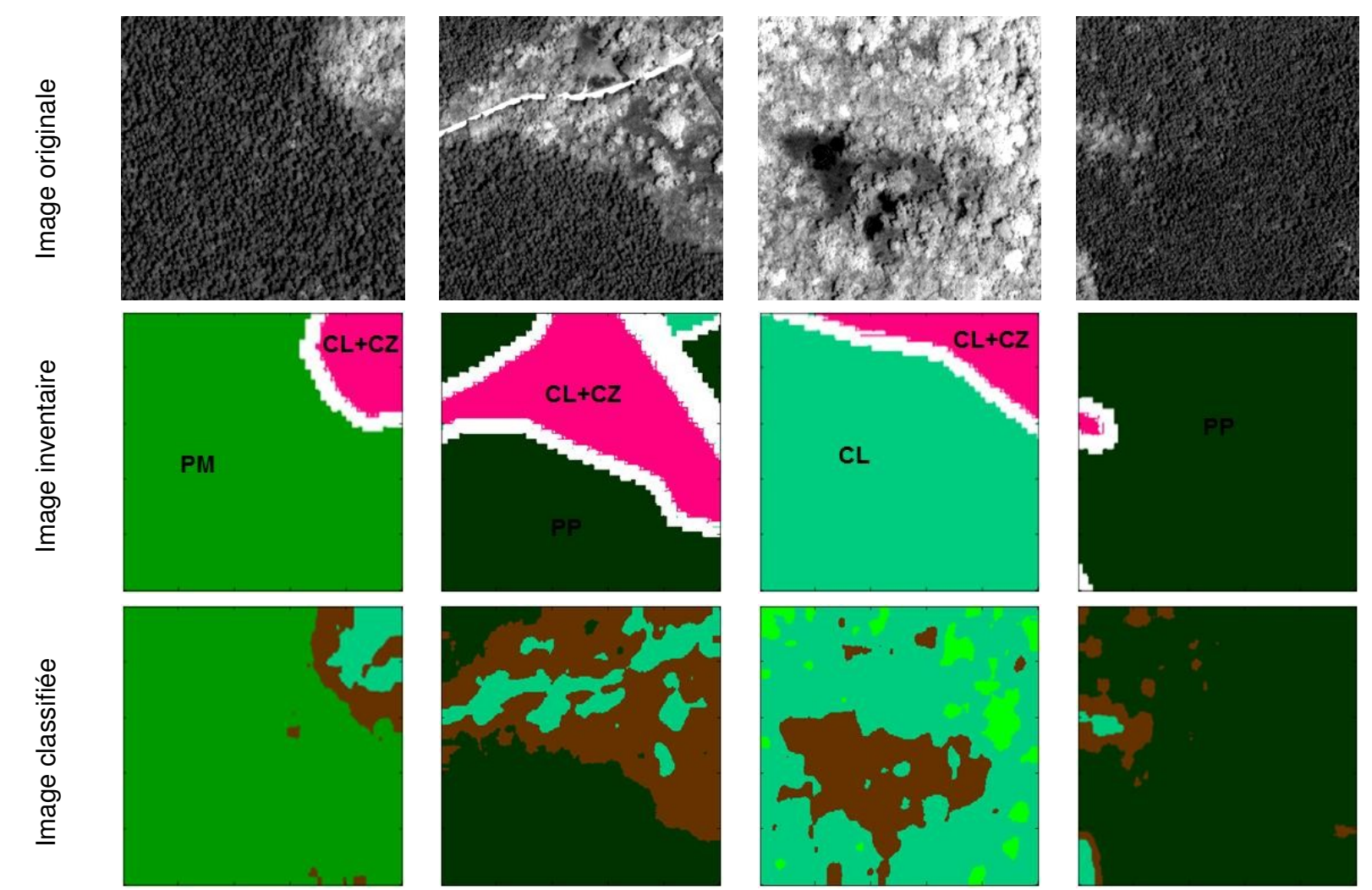

Kappa

92,43

77,34

79,19

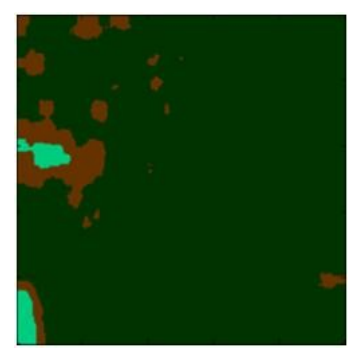

66,09

89,07

88,08

98,18

TABLE 9 : Sous-images, leurs images inventaires correspondantes, et leurs résultats de classification. 
Clausi D. A., 2002. An analysis of cooccurrence texture statistics as a function of grey level quantization. Canadian Journal of Remote Sensing, 28(1) :45-62.

Diou C., 2000. Contribution à l'intégration sur silicium de la transformée en ondelettes Application au traitement d'images. PhD thesis, University Montpellier 2, France.

Djema L., Boumghar F., Bachir Y. A., 2012. Parallélisation du filtre de Gabor pour l'extraction des caractéristiques des textures d'image. Proceedings of the IEEE Conference of Science of Electronics, Technologies of Informations and Telecommunications, Sousse, Tunisie, $1-6$.

Gabor D., 1946. Theory of communication. Part 1 : The analysis of information. Proceedings of the Institution of Electrical Engineers, 93(26) :429-457.

Galloway M. M., 1975. Texture analysis using gray level run lengths. Computer Graphics and Image Processing, 4(2) :172-179.

Grazzini J., 2003. Analyses multi-échelle et multifractale d'images météorologiques: Application à la détection de zones précipitantes. PhD thesis, University Marne-laVallée, France.

Haralick R. M., Shanmugam K., Dinstein I., 1973. Textural features for image classification. IEEE Transaction on Systems, Man and Cybernetics, 3(6) :610-621.

Krim J., Indekeu J. O., 1993. Roughness exponents : A paradox resolved. Physical Review E, 48(2) :1576-1578.

Laws K.I., 1980. Textured Image Segmentation. PhD thesis, University of Southern California, California.

Lefebvre A., 2011. Contribution de la texture pour l'analyse d'images à THR spatiale : Application à la détection de changement en milieu périurbain. PhD thesis, University Rennes 2, France.

Macqueen J.B., 1967. Some Methods for classification and Analysis of Multivariate Observations. Proceedings of 5th Berkeley Symposium on Mathematical Statistics and Probability, Berkeley, California, 281-297.

Marceau D.J., Howarth P.J., Dubois J.M., Gratton D.J., 1990. Evaluation of the grey-level co-occurrence matrix method for land-cover classification using SPOT imagery. IEEE Transactions on Geoscience and Remote Sensing, 28(4) :513-519.

Messier N., Cavayas F., André P., 2001. Cartographie des occupations du sol en milieu urbain, à partir d'images satellites de haute résolution spatiale : le cas de Beijing. Proceedings of the 23rd Canadian Symposium on Remote Sensing, Quebec City, Canada, 829-834.

Ojala T., Pietikainen M., Harwood D., 1996. A comparative study of texture measures with classification based on featured distributions. Pattern Recognition, 29(1) :51-59.

Penatti O., Valle E., Torres R., 2012. Comparative study of global color and texture descriptors for web image retrieval. Journal Of Visual Communication And Image Representation, 23(2) :359-380.

Porebski A., 2010. Sélection d'attributs de texture couleur pour la classification d'images : Application à l'identification de défauts sur les décors verriers imprimés par sérigraphie. PhD thesis, University Lille 1, France.

Prasetiyo, Khalid M., Yusof R., Meriaudeau F., 2010. A comparative study of feature extraction methods for wood texture classification. Proceedings of the IEEE 6th International Conference on Signal-Image Technology and Internet-Based Systems, Kuala Lumpur, Malaysia, 23-29.

Rellier G., Descombes X., Falzon F., Zerubia J., 2002. Analyse de texture hyper-spectrale par modélisation markovienne. Research Report, INRIA, 4479.

Saint-Onge B., 1994. L'apport de la texture des images numériques de haute résolution à la cartographie forestière automatisée. PhD thesis, University of Montreal, Canada.

Tahiri Alaoui M., Niang M, Farssi S. M., 2006. Classification des images de textures en utilisant une approche multifractale. Journal of Sciences, 6(2) :116-124.

Tan X., Triggs B., 2010. Enhanced local texture feature sets for face recognition under difficult lighting conditions. IEEE Transactions on Image Processing, 19(6) :1635-1650.

Turiel A., Parga N., 2000. The multifractal structure of contrast changes in natural images : from sharp edges to textures. Neural Computation, 12(4):763-793.

Turiel A., Parga N., 2000. Multifractal wavelet filter of natural images. Physical Review Letters, 85(15) :33253328.

Unser M., 1986. Sum and difference histograms for texture classification. IEEE Transactions on Pattern Analysis and Machine Intelligence, 8(1) :118-125.

Xia Y., Feng D., Zhao R., Zhang Y., 2010. Multifractal signature estimation for textured image segmentation. Pattern Recognition Letters, 31(2) :163-169. 